\title{
Assessing the Stroke-Specific Quality of Life for Outcome Measurement in Stroke Rehabilitation: Minimal Detectable Change and Clinically Important Difference
}

\author{
Keh-chung Lin ${ }^{1,2}$, Tiffany Fu' ${ }^{1}$ Ching-yi Wü ${ }^{3 *}$ Ching-ju Hsieh ${ }^{4}$
}

\begin{abstract}
Background: This study was conducted to establish the minimal detectable change (MDC) and clinically important differences (CIDs) of the physical category of the Stroke-Specific Quality of Life Scale in patients with stroke.

Methods: MDC and CIDs scores were calculated from the data of 74 participants enrolled in randomized controlled trials investigating the effects of two rehabilitation programs in patients with stroke. These participants received treatments for 3 weeks and underwent clinical assessment before and after treatment. To obtain testretest reliability for calculating MDC, another 25 patients with chronic stroke were recruited. The MDC was calculated from the standard error of measurement (SEM) to indicate a real change with 95\% confidence for individual patients $\left(\mathrm{MDC}_{95}\right.$ ). Distribution-based and anchor-based methods were adopted to triangulate the ranges of minimal CIDs. The percentage of scale width was calculated by dividing the MDC and CIDs by the total score range of each physical category. The percentage of patients exceeding $\mathrm{MDC}_{95}$ and minimal CIDs was also reported.
\end{abstract}

Results: The $\mathrm{MDC}_{95}$ of the mobility, self-care, and upper extremity (UE) function subscales were 5.9, 4.0, and 5.3 respectively. The minimal CID ranges for these 3 subscales were 1.5 to 2.4, 1.2 to 1.9, and 1.2 to 1.8. The percentage of patients exceeding $\mathrm{MDC}_{95}$ and minimal CIDs of the mobility, self-care, and UE function subscales were $9.5 \%$ to $28.4 \%, 6.8 \%$ to $28.4 \%$, and $12.2 \%$ to $33.8 \%$, respectively.

Conclusions: The change score of an individual patient has to reach 5.9, 4.0, and 5.3 on the 3 subscales to indicate a true change. The mean change scores of a group of patients with stroke on these subscales should reach the lower bound of CID ranges of 1.5 (6.3\% scale width), 1.2 (6.0\% scale width), and 1.2 (6.0\% scale width) to be regarded as clinically important change. This information may facilitate interpretations of patient-reported outcomes after stroke rehabilitation. Future research is warranted to validate these findings.

\section{Background}

Although the stroke mortality rate has been declining [1], the estimated prevalence rate of stroke-related disability is about 331 per 100,000 [2]. Stroke disability and morbidity cause reduced quality of life (QOL) among stroke survivors [3]. The greater the disability, the lower the QOL is [4]. With ongoing rehabilitation, however,

\footnotetext{
* Correspondence: cywucywu1@gmail.com

${ }^{3}$ Department of Occupational Therapy and Graduate Institute of Clinical Behavioral Science, Chang Gung University, 259 Wenhua 1st Road, Taoyuan, Taiwan

Full list of author information is available at the end of the article
}

improvements in functional status are possible [5] and contribute to increase QOL for stroke survivors. Therefore, the assessment of stroke rehabilitation should include disability and QOL domains, which are influenced by the disease [6-9].

Generic QOL instruments such as the Medical Outcomes Study Short-Form 36-item survey (SF-36) may underestimate the effect of stroke [10]; therefore, disease-specific tools are considered more helpful in providing information about the difficulties that patients with stroke may experience $[7,11]$. Because the 
information from the patients' perspective on the consequences of disease and the therapeutic benefits is considered critical in the evaluation of health care, patient-reported outcome measures have been used to supplement clinical decisions made from physicianbased outcome measures [12]. Of the stroke-specific scales, the Stroke-Specific Quality of Life Scale (SS-QOL) [13], in addition to the Stroke Impact Scale version 3.0 (SIS 3.0) [14], is the most comprehensive [15] and frequently used patient-reported outcome measure [16-19].

The SS-QOL is a self-report questionnaire consisting of 49 items in the 12 domains of energy, family roles, language, mobility, mood, personality, self-care, social roles, thinking, upper extremity (UE) function, vision, and work/productivity. The domains are scored separately, and a total score is also provided. The psychometric properties of the SS-QOL have been validated in patients with ischemic stroke and intracerebral hemorrhage $[10,18,20]$. In patients with subarachnoid hemorrhage, the 12 SS-QOL domains and the total score demonstrated good internal consistency [21]. The SSQOL items also have acceptable agreement with the categories of the International Classification of Functioning, Disability, and Health, which indicates that the SS-QOL covers multidimensional components meaningful for patients with stroke [22]. The clinical utility of the SS-QOL remains understudied, however, and several clinimetric properties, such as the minimal detectable change (MDC) and the clinically important difference (CID) of the SS-QOL, have not yet been investigated. This information helps inform clinical decision making on the discontinuation or alteration of a treatment program that aims to improve patients' physical function.

The MDC is the smallest change that can be detected by the instrument beyond measurement error. The CID is a related concept that shows how much change can be deemed as clinically important [23]. That is, CID is the threshold score that a group of patients perceive as noticeable. The MDC and CID facilitate the interpretation of treatment outcomes. For example, the study by Lin et al [24] reported that a true change in the SIS mobility subscale that occurs after rehabilitation needs to show an increase of at least 15.1 points or the change is likely due to an error in the measurement.

In some instances, CID scores do not exceed the MDC scores but still convey information about whether a patient group experienced a clinically important change. In the study of Plummer et al [25], for example, the improvement of $0.11 \mathrm{~m} / \mathrm{s}$ in gait speed was lower than the measurement error of $0.17 \mathrm{~m} / \mathrm{s}$ reported by Evans et al [26], indicating that the improvement of gait speed might not be real and beyond measurement error. However, the change of $0.11 \mathrm{~m} / \mathrm{s}$ gait speed in the Plummer et al [25] study indicated that this patient group improved from the category of physiologic ambulatory to that of full-time home ambulatory, according to the walking categories developed by Perry et al [27]. Without these important benchmarks against which the clinical interpretation is based, clinicians may make erroneous conclusions about the effect of a treatment.

Therefore, this study sought to establish the MDC and CID score estimates of the SS-QOL subscales and assess the proportion of patients' change scores on the SSQOL subscales that exceeded the MDC and CID in a cohort of patients with stroke who received rehabilitation therapies.

\section{Methods}

\section{Participants}

The study protocol consisted of 2 parts. First, the CIDs data were obtained from participants in randomized controlled trials investigating the effects of 2 upper limb training programs $[28,29]$. These participants were consecutively screened and recruited from 4 stroke rehabilitation units. Of 126 patients receiving the intervention in these 2 randomized controlled trials, 74 completed the SS-QOL and were included in the present study. The second part of the study is related to MDC. To obtain the test-retest reliability for calculating $\mathrm{MCD}$ [30], we recruited 25 patients with chronic stroke from another independent sample.

The inclusion and exclusion criteria for these 2 samples (74 patients for part 1 and 25 samples for part 2) were the same. The inclusion criteria of this study include: first-ever stroke, at least 6 months' poststroke, demonstration of Brunnstrom stage III or higher for the proximal part of the affected upper limb [31], no serious cognitive deficits (score $>24$ on the Mini Mental-State Exam) [32], and no excessive spasticity at any joint of the upper limb (score of $\leq 2$ on the Modified Ashworth Scale) [33].

Excluded were patients with physician-determined major medical problems and severe aphasia that could potentially confound the study results. This study was approved by Chang Gung Memorial Hospital Human Research Ethics Board (96-0252B) and National Taiwan University Hospital Research Ethics Committee (200903080R), and all participants signed the informed consent forms.

\section{Interventions and Procedures}

Only the 74 participants received 1 of the 3 rehabilitation programs: bilateral arm training (BAT), distributed constraint-induced therapy (CIT), or conventional rehabilitation. Therapy in the BAT group emphasized simultaneous movement of the affected and the unaffected upper limb. The distributed CIT group focused on restriction of movement of the unaffected limb and 
intensive training of the affected limb. The conventional rehabilitation group focused on neurodevelopment techniques with an emphasis on functional task practice, when possible. The interventions were provided at the participating hospitals under the supervision of 3 certificated occupational therapists. The raters were blinded to the participant group and trained to properly administer the outcome measures. Rater competence was assessed by a senior certified occupational therapist. The same rater administered the SS-QOL evaluation at the 2 different time points (baseline and after the 3-week intervention) for each participant.

\section{Outcome Measure}

The SS-QOL contains 12 subscales (as detailed earlier) with a total of 49 items derived from a series of focused interviews with 34 ischemic stroke survivors [13]. Scoring of the SS-QOL concerns the past week and is rated on a 5-point Likert scale. Response options are scored as 5 ("no help needed/no trouble at all/strongly disagree"), 4 ("a little help/a little trouble/moderately disagree"), 3 ("some help/some trouble/neither agree nor disagree"), 2 ("a lot of help/a lot of trouble/moderately agree"), and 1 ("total help/could not do it at all/strongly agree"). The SS-QOL provides domain scores and a summary score, with higher scores indicating better function. The test-retest reliability, internal consistency, construct, and convergent validity of the SS-QOL have been ascertained in patients with stroke $[10,18,21]$.

Furthermore, the Chinese version of SS-QOL demonstrated adequate Rasch separation reliability and unidimensionality [34]. Because the intervention focused on the rehabilitation of the paretic arm and the improvement of daily functioning, the CID scores based on physical-related subscales directly reflect the benefit of motor intervention. As a result, we only reported the $\mathrm{MDC}_{95}$ and CID of the SS-QOL subscales that are related to physical function, including mobility, self-care, and UE function [35].

\section{Data Analysis}

\section{Estimation of MDC}

The MDC is calculated by multiplying the standard error of measurement (SEM) by 1.96 to correspond to the $95 \%$ confidence interval and the square root of 2 to adjust for sampling from 2 different measurements [36]. The SEM is estimated as the pooled standard deviation (SD) of test-retest assessments multiplied by the square root of $(1-r)$, where $r$ is the intraclass correlation coefficient (ICC) [37]. The ICC, a kind of test-retest reliability, was determined using a set of independent data from 25 patients in whom the SS-QOL assessment was conducted 2 weeks apart. The ICC was calculated using a 2-way mixed effect model, with a consistency coefficient. $\mathrm{MDC}_{95}$ means one can be $95 \%$ confident that a change score equal to or exceeding this threshold is true and reliable and not just measurement error [23].

\section{Estimation of CID}

The distribution-based and the anchor-based approaches were both used to determine the CIDs of the subscales of the SS-QOL. The distribution-based CID estimate was determined using the between-participant baseline SD and the SEM within-participant methods to estimate the CID scores [38]. An effect size is a standardized measure of change over time and represents individual change in terms of the number of pretest SDs. For example, an effect size of 0.5 indicates an increase of 0.5 SD. Cohen [39] has provided benchmarks that serve to guide the interpretations of effects size. According to Ringash et al [40], CIDs are generally close to an effect size of 0.2 , and an effect size of 0.5 represents humans' limitation in discrimination [41]. We chose 0.5 SD units to estimate the minimal threshold of CIDs. The SD varies with the heterogeneity of the sample and does not take patient variability of change into consideration. The SEM, which simultaneously incorporates both the sample's reliability and variability into the formula and is relatively sample-independent, is used as another indicator of minimal CID [37].

The anchor-based CID estimate was calculated as the mean change score on each SS-QOL subscale, corresponding to patients who perceived overall increased recovery of $10 \%$ to $15 \%$ in the Stroke Impact Scale (SIS). We chose SIS as the anchor during the calculation of CID estimates because the overall recovery ratings on SIS directly reflect the participant's viewpoint on the health-related recovery $[42,43]$.

Although there is no defined range of the change score as to the determination of the CID group, several previous studies have found the smallest change score of $10 \%$ on the $100-\mathrm{mm}$ visual analog scale (VAS) of quality of sleep [44], $11 \%$ on the 100-point Pediatric Evaluation of Disability Inventory (PEDI) [45], and 15\% on the 100-mm VAS of back pain [46]. In addition, Duncan et al [47] suggested the clinically meaningful improvement of the SIS global rating scale is within $10 \%$ to $15 \%$ change. Therefore, patients in the current study were classified into the CID group if a $10 \%$ to $15 \%$ change was documented on their perceived overall recovery from pretreatment to posttreatment and were considered as having experienced a clinically important change.

Furthermore, to assess the extent of patients' changes after interventions detected by the SS-QOL subscales, the percentage of scale width was calculated by dividing the MDC and CIDs by the total score range of each physical category. For example, the score range of the mobility subscale was from 6 to 30 , the total score 
range of the mobility subscale was 24 . In addition, the proportions of patients with change scores greater than the $\mathrm{MDC}_{95}$ values and the minimal threshold of CID estimates were calculated.

\section{Results}

Table 1 presents the demographic and clinical characteristics of the 74 patients enrolled in this study as well as the additional 25 patients from the independent sample for calculating test-retest reliability. All characteristics were comparable between these 2 samples, and there were no preexisting differences between the 2 samples on any of the variables.

As indicated in Table 2 the $\mathrm{MDC}_{95}$ of the mobility, self-care, and UE function subscales were 5.9 (24.6\% scale width), 4.0 (20.0\% scale width), and 5.3 (26.5\% scale width), respectively. According to anchor-based and distribution-based methods, we suggest the respective group-level CIDs for these 3 subscales are in range of 1.5 to 2.4 ( $6.3 \%$ to $10 \%$ scale width), 1.2 to 1.9 (6.0\% to $9.5 \%$ scale width), and 1.2 to $1.8(6.0 \%$ to $9.0 \%$ scale width) for the mobility, self-care, and UE function subscales, respectively. As reported in Table 3 an estimated $9.5 \%, 6.8 \%$, and $12.2 \%$ of the patients had a positive change that exceeded the $\mathrm{MDC}_{95}$ of the mobility, selfcare, and UE function subscales, and $28.4 \%, 28.4 \%$, and $33.8 \%$ of patients' change scores exceeded the lower bound of CID ranges of the mobility, self-care, and UE function subscales, respectively.

$\begin{aligned} & \text { Table } \mathbf{1} \text { Demographic and baseline clinical characteristics } \\
& \text { of the participants }\end{aligned}$
\begin{tabular}{llll}
\hline Characteristic & $\mathbf{n}^{\mathbf{a}}=\mathbf{7 4}$ & $\mathbf{n}^{\mathbf{b}}=\mathbf{2 5}$ & $\mathbf{P}$ \\
\hline Age, mean (SD) y & 57.1 & 52.9 & $0.89^{c}$ \\
& $(11.7)$ & $(11.2)$ & \\
Gender, Male/Female, No. & $52 / 22$ & $17 / 8$ & $1.00^{d}$ \\
Months after stroke, mean (SD) & 18.1 & 15.5 & $0.82^{c}$ \\
& $(16.4)$ & $(12.8)$ & \\
Side of stroke, Right/Left, No. & $38 / 36$ & $16 / 9$ & $0.25^{d}$ \\
Stroke subtype, Hemorrhagic/lschemic, No. & $28 / 46$ & $12 / 13$ & $0.49^{d}$ \\
Brunnstrom stage of proximal UE, median & $4.5(3-6)$ & $4(3-6)$ & $0.61^{d}$ \\
(range) & & & \\
Fugl-Meyer Assessment UE scores, mean & 44.0 & 40.8 & $0.23^{c}$ \\
(SD) & $(13.1)$ & $(14.1)$ & \\
Mini Mental-State Exam scores, mean (SD) & $27.5(2.3)$ & $26.6(2.8)$ & $0.23^{c}$ \\
Intervention, No. & & & \\
$\quad$ Bilateral Arm Training & 22 & & \\
$\quad$ Constraint-Induced Therapy & 16 & & \\
$\quad$ Conventional Rehabilitation. & 36 & & \\
\hline
\end{tabular}

Abbreviations: SD, standard deviation; UE, upper extremity.

${ }^{a}$ The participants used for estimating clinically important differences; ${ }^{b}$ The participants used for estimating the test-retest reliability; ${ }^{c}$ The two-sample t-test, 2-tailed; ${ }^{\mathrm{d}}$ Chi-square.

\section{Discussion}

To the best of our knowledge, this is the first study to determine the MDC and CID scores of the SS-QOL subscales that can be used to differentiate patients treated with stroke rehabilitation who experience real improvement and clinically meaningful change from those who do not. Our findings suggest that a patient's change score has to reach 5.9, 4.0, and 5.3 on the mobility, self-care, and UE function subscales to indicate a true change. That is, when the change scores between the patient's 2 measurements (e.g., baseline and followup) reach $24.6 \%, 20.0 \%$, and $26.5 \%$ of the scale width on the mobility, self-care, and UE function subscales, the clinicians may interpret the changes in that patient as true and reliable, given the $95 \%$ confidence level.

There is no universally accepted standard for determining the CID [48-52]. An integrated system for defining CID is recommended that combines anchor-based and distribution-based methods [48]. The value and limitations of anchor-based and distribution-based methods in estimating CID have been recognized. The anchorbased approach emphasizes the primacy of a patient's perspective, but anchor-based CID scores may vary with demographic characteristics such as age [49]. Although the distribution-based CID scores are easy to generate, these SD-based scores are associated with some bias due to sample heterogeneity [38]. As a result, a number of recent clinical reports have advocated an approach that combines the anchor-based and distribution-based methods to refine the range of CID [24,50,51].

Using a 1 SEM distribution-based approach, we found that the CIDs for the mobility, self-care, and UE function subscales are 1.7 (7.1\% scale width), 1.2 (6.0\% scale width), and 1.3 (6.5\% scale width), respectively. The SEM incorporates a sample's variability and the reliability of the instrument. Several previous studies have shown that $1 \mathrm{SEM}$ is close to the estimate of CID [53-56]. Despite being theoretically constant [56], the SEM may become larger with a low reliability [57].

Furthermore, the CID scores using 1 SEM would be always less than the MDC values mathematically. Therefore, values of 0.5 SD were calculated as supportive information for determining the CID. On the basis of the $0.5 \mathrm{SD}$ approach, we found that the CID scores for the subscales were 2.4 ( $10 \%$ scale width) for mobility, 1.9 (9.5\% scale width) for self-care, and 1.8 (9\% scale width) for UE function.

The CID values produced by the anchor-based method were 1.5 (6.3\% scale width) for mobility, $1.3(6.5 \%$ scale width) for self-care, and $1.2(6.0 \%$ scale width) for UE function. These estimates were comparable with those obtained from the distribution-based approaches. Because a cutoff threshold of the group-level CID may 
Table 2 Threshold values of the MDC $_{95}$ and clinically important difference (CID) of the SS-QOL subscales

\begin{tabular}{|c|c|c|c|c|c|c|}
\hline \multirow[t]{2}{*}{ Subscale } & \multirow[t]{2}{*}{ Score range } & \multirow[t]{2}{*}{ ICC $(95 \% \mathrm{Cl})$} & \multirow[t]{2}{*}{$M{ }^{25}$ (\% scale width) } & \multicolumn{2}{|c|}{ Distribution-based CID } & \multirow{2}{*}{$\begin{array}{l}\text { Anchor-based CID } \\
\text { (\% scale width) }\end{array}$} \\
\hline & & & & 0.5 SD (\% scale width) & 1 SEM (\% scale width) & \\
\hline Mobility & $6-30$ & $0.84(0.63,0.93)$ & $5.9(24.6 \%)$ & $2.4(10 \%)$ & $1.7(7.1 \%)$ & $1.5(6.3 \%)$ \\
\hline Self-care & $5-25$ & $0.88(0.73,0.95)$ & $4.0(20.0 \%)$ & $1.9(9.5 \%)$ & $1.2(6.0 \%)$ & $1.3(6.5 \%)$ \\
\hline UE function & $5-25$ & $0.88(0.72,0.95)$ & $5.3(26.5 \%)$ & $1.8(9.0 \%)$ & $1.3(6.5 \%)$ & $1.2(6.0 \%)$ \\
\hline
\end{tabular}

Abbreviations: $\mathrm{Cl}$, confidence interval; ICC, intraclass correlation coefficient; $\mathrm{MDC}_{95}$, minimal detectable change at $95 \%$ confidence; SD, standard deviation; SEM, standard error of measurement; UE, upper extremity.

potentially undermine the clinical interpretation of trial data [58], we reported ranges rather than a single value. We found the CID ranges were 1.5 to 2.4 for mobility, 1.2 to 1.9 for self-care, and 1.2 to 1.8 for UE function. That is, patients with stroke who achieve mean scores in the ranges of $6.3 \%$ to $10.0 \%, 6.0 \%$ to $9.5 \%$, and $6.0 \%$ to $9.0 \%$ of the scale width on the mobility, self-care, and UE function subscales are likely to have clinically meaningful change in these domains.

Of note, there is a concern about the differences between group and individual clinical importance [59]. Average effects across a group may not be meaningful to the individual patient. Group-derived CID values are suitable to interpret the results of clinical trials or group studies, but they are often directly applied to interpret the individual's change [59]. For individual-level use, it may be reasonable to expect that the MDC would be less than or equal to the minimal CID. However, some researchers have suggested that this is not always the case $[24,60]$, which is also consistent with our current findings. When the MDC exceeds the minimal CID, the change score reaching a CID does not mean that patients have exceeded the measurement error, and both values are suggested to be considered in clinical decision making [61].

Taking our cohort sample of stroke rehabilitation as an example, the mean change scores on the mobility, self-care, and UE function subscales were $3.5,2.8$, and 4.1 points, which exceeded the minimal CID ranges. This indicated that the improvements achieved after rehabilitative therapies in this cohort were meaningful to the patients. A mean change score of 1.2 on the selfcare subscale in a previous study of the Chronic Disease

Table 3 Number of participants who met the criteria of the $\mathrm{MDC}_{95}$ and clinically important difference (CID)

\begin{tabular}{|c|c|c|c|c|}
\hline \multirow[t]{2}{*}{ Subscale } & \multirow[t]{2}{*}{$\mathrm{MDC}_{95}$} & \multicolumn{2}{|c|}{ Distribution-based CID } & \multirow[t]{2}{*}{ Anchor-based CID } \\
\hline & & 0.5 SD & 1 SEM & \\
\hline & No. (\%) & No. (\%) & No. (\%) & No. (\%) \\
\hline Mobility & $7(9.5 \%)$ & $15(20.3 \%)$ & $21(28.4 \%)$ & $21(28.4 \%)$ \\
\hline Self-care & $5(6.8 \%)$ & $21(28.4 \%)$ & $21(28.4 \%)$ & $21(28.4 \%)$ \\
\hline UE function & $9(12.2 \%)$ & $25(33.8 \%)$ & 25 (33.8\%) & $25(33.8 \%)$ \\
\hline
\end{tabular}

Abbreviations: $\mathrm{MDC}_{95}$, minimal detectable change at 95\% confidence; SD, standard deviation; SEM, standard error of measurement; UE, upper extremity.
Self-Management course [17] was reported to achieve statistical significance. This improvement at the group level failed to achieve the lower bound of the minimal CID range established by our current study, which may weaken the validity of the study conclusion about the effect of the self-management education on the quality of self-care after stroke.

Although the validity of a self-rated global assessment scale has been criticized for its "retrospective bias" $[50,62,63]$, we recognized that clinical interpretation of the MDC and CID scores would be enhanced if a patient-driven anchor were included in the study design. Therefore, the reliable-change approach, as proposed by Davidson and Keating [64], was adopted to expand the clinical application of the $\mathrm{MDC}_{95}$ and CID established by the current study. The reliable-change approach addresses the question about the proportion of patients exceeding the threshold of MDC and CID. The concept is similar to the event rate, which represents the number of people in whom an event is observed [65]. For example, the event rate is $40 \%$ if 40 of 100 patients experience an adverse event such as side effect. On the basis of our results, $9.5 \%, 6.8 \%$, and $12.2 \%$ of patients achieved functional improvement beyond measurement error on the mobility, self-care, and UE function subscales. The greatest proportion of patients that exceeded the lower bound of the minimal CID was observed for the UE function subscale (33.8\%), followed by the selfcare $(28.4 \%)$ and mobility (28.4\%) subscales. According to Schmitt and Fabio [66], the better the responsiveness of a scale is, the greater the numbers of patients who will exceed the minimal change criteria. Thus, the UE function subscale appears the most responsive subscale among those in the physical category of the SS-QOL for the patients of this study. Because the focus of the rehabilitation used in the current study was on the functional recovery of the paretic arm, it is also possible that the intervention effect was responsible for the relatively greater proportion of patients who exceeded the MDC and CID of the UE function subscale. Further research using larger samples is needed to validate the findings.

It is important to note that the participants included in this study were assigned to receive different treatment programs; thus, the variance in the change scores might 
have varied among the different treatment groups. Additional analyses of the CIDs for each intervention group showed that the differences in CID values represented by 1 SEM between the participants of each intervention group and the overall participants were less than 0.6 points in the mobility subscale (each intervention participants: 1.3-2.3; vs. overall participants: 1.7 ) and 0.4 point in the self-care (1.1-1.6 vs. 1.2) and UE function subscales (1.1-1.7 vs. 1.3); and the differences in CID values represented by $0.5 \mathrm{SD}$ between each intervention group participants and the overall participants were less than 0.7 points (mobility: $1.8-3.1$ vs. 2.4 , self-care: 1.6-2.4 vs. 1.9, and UE function: 1.6-2.5 vs. 1.8). Generally speaking, the CID values in each intervention group are arguably close enough to allow collapse of data from all intervention groups into one group for analysis in each subscale. Given the above information and the fact that the same amount of treatment duration and intensity were used across the different treatment programs, we felt the method of collapsing the data from various intervention groups would be justifiable. For example, some recent studies $[67,68]$ have combined the data from different intervention groups for clinimetric analyses.

The current investigation has some limitations that warrant consideration when interpreting and generalizing the study findings. First, the generalizability of the current findings might be limited. Because we only included patients from departments of rehabilitation with the demonstration of Brunnstrom stage III or higher for the affected UE, the current findings may not be suitable for stroke patient at a Brunnstrom stage of less than III. In addition, some patients were excluded from the current investigation due to cognitive difficulties. To increase the external validity of the results of this study, it is warranted to recruit a wider sample of patients with stroke with differing levels of motor impairment and cognitive difficulty.

Second, because of the relevance of proxy reports for QOL outcome evaluations, particularly in patients with stroke with language impairments [69], there is a need for extended research on the clinimetric properties of the proxy version of the SS-QOL to establish the minimal significant change perceived by the proxies.

Third, although patients who have received different treatment programs with the same treatment duration are often pooled together for clinimetric analysis of the outcome measures $[67,68]$, further research is needed that may investigate the MDC and CID of the SS-QOL for specific interventions based on larger samples to provide further insights into the clinimetric properties of the SS-QOL in specific contexts.

Finally, there are potential clinimetric differences in patient-reported QOL outcomes due to the modes of administration [70]; thus, further research may study clinimetric attributes of the SS-QOL administered in different modes, such as paper-and-pencil administration vs. telephone interviews vs. Web-based electronic data collection.

\section{Conclusions}

In addition to providing information about the psychometric properties of the SS-QOL subscales, the preliminary results of the MDC and CID of the SS-QOL subscales established by this study facilitate the interpretation of the change scores observed in patients with stroke receiving rehabilitation therapies. We found that a patient's change score has to reach 5.9 (24.6\% scale width) on the SS-QOL mobility, 4.0 (20.0\% scale width) on the self-care, and $5.3(26.5 \%$ scale width) on the UE function subscales to indicate a true and reliable improvement. If the mean change scores for the SS-QOL subscales within a stroke patient group are 1.5 to 2.4 (6.3\% to $10 \%$ scale width) for mobility, 1.2 to 1.9 (6.0\% to $9.5 \%$ scale width) for self-care, and 1.2 to 1.8 (6.0\% to $9.0 \%$ scale width) for UE function, the changes may be considered clinically important. According to the proportions of patients who met the MDC and CID criteria, the UE function subscale seems more responsive than the mobility and self-care subscales for the patients of this study. This may be related to the nature of the rehabilitation therapies involved in our research (i.e., physical interventions that emphasized UE function). Findings of the present study warrant further study based on larger samples involving different types of stroke rehabilitation programs.

\section{Acknowledgements}

This research was supported in part by grants from the National Science Council (NSC-97-2314-B-002-008-MY3, NSC-97-2314-B-182-004-MY3, NSC-972811-B-002-101, and NSC-98-2811-B-002-073) and the National Health Research Institutes (NHRI-EX99-9920PI and NHRI-EX99-9742PI).

\section{Author details}

${ }^{1}$ School of Occupational Therapy, College of Medicine, National Taiwan University, 17, F4, Xu Zhou Road, Taipei, Taiwan. ${ }^{2}$ Division of Occupational Therapy, Department of Physical Medicine and Rehabilitation, National Taiwan University Hospital, 7 Chung-shan South Road, Taipei, Taiwan. ${ }^{3}$ Department of Occupational Therapy and Graduate Institute of Clinical Behavioral Science, Chang Gung University, 259 Wenhua 1st Road, Taoyuan, Taiwan. ${ }^{4}$ Institute of Biophotonics, National Yang-Ming University and Department of Ophthalmology, Taipei City Hospital-Heping Branch, Taipei, Taiwan.

\section{Authors' contributions}

$\mathrm{KCL}$ conceived the study, participated in its design and coordination, and helped to draft the manuscript. TF participated in the design of the study, performed the statistical analysis, and participated in the writing of the manuscript. CYW contributed to secure the research funding, designed and conducted the study, and participated in the data interpretation. $\mathrm{CJH}$ contributed to the revision of the manuscript. All authors read and approved the final manuscript. 


\section{Competing interests}

The authors declare that they have no competing interests.

Received: 15 June 2010 Accepted: 19 January 2011

Published: 19 January 2011

\section{References}

1. Lavados PM, Hennis AJM, Fernandes JG, et al: Stroke epidemiology, prevention, and management strategies at a regional level: Latin America and the Caribbean. Lancet Neurol 2007, 6:362-372.

2. Mar J, Sainz-Ezkerra M, Moler-Cuiral JA: Calculation of prevalence estimates through differential equations: application to stroke-related disability. Neuroepidemiology 2008, 31:57-66.

3. Muus I, Petzold M, Ringsberg KC: Health-related quality of life among Danish patients 3 and 12 months after TIA or mild stroke. Scand J Caring Sci 2010, 24(2):211-8.

4. Aprile I, Piazzini DB, Bertolini C, et al: Predictive variables on disability and quality of life in stroke outpatients undergoing rehabilitation. Neurol Sci 2006, 27:40-46.

5. Dhamoon MS, Moon YP, Paik MC, et al: Long-term functional recovery after first ischemic stroke: The Northern Manhattan study. Stroke 2009, 40:2805-2811.

6. Bowling A: Measuring Health: A Review of Quality of Life Measurement Scales. 3 edition. Maidenhead, Berkshire, England: Open University Press; 2005.

7. Carod-Artal FJ, Egido JA: Quality of life after stroke: the importance of a good recovery. Cerebrovasc Dis 2009, 27:204-214.

8. Graham A: Measurement in stroke: activity and quality of life. In Recovery after Stroke. Edited by: Barnes MP, Dobkin BH, Bogusslavsky J. New York: Cambridge University Press; 2005:135-160.

9. Kissela B: The value of quality of life research in stroke. Stroke 2006, 37:1958-1959.

10. Lima RCM, Teixeira-Salmela LF, Magalhaes LC, Gomes-Neto M: Psychometric properties of the Brazilian version of the Stroke Specific Quality of Life Scale: application of the Rasch model. Rev Bras Fisioter 2008, 12:149-156.

11. Noble AJ, Schenk T: Which variables help explain the poor health-related quality of life after subarachnoid hemorrhage? A meta-analysis. Neurosurgery 2010, 66:772-783.

12. Hobart JC, Riazi A, Lamping DL, Fitzpatrick R, Thompson AJ: Improving the evaluation of therapeutic interventions in multiple sclerosis: development of a patient-based measure of outcome. Health Technol Assess 2004, 8(9).

13. Williams $L S$, Weinberger $M$, Harris $L E$, Clark DO, Biller J: Development of a Stroke-Specific Quality of Life Scale. Stroke 1999, 30:1362-1369.

14. Duncan PW, Bode RK, Lai SM, Perera S: Rasch analysis of a new strokespecific outcome scale: the Stroke Impact Scale. Arch Phys Med Rehabil 2003, 84:950-963.

15. Salter KL, Moses MB, Foley NC, Teasell RW: Health-related quality of life after stroke: what are we measuring? Int J Rehabil Res 2008, 31:111-117.

16. Chou PC, Chu HY, Lin JG: Effects of electroacupuncture treatment on impaired cognition and quality of life in Taiwanese stroke patients. $J$ Altern Complement Med 2009, 15:1067-1073.

17. Kendall E, Catalano T, Kuipers P, Posner N, Buys N, Charker J: Recovery following stroke: the role of self-management education. Soc Sci Med 2007, 64:735-746.

18. Muus I, Williams LS, Ringsberg KC: Validation of the Stroke Specific Quality of Life scale (SS-QOL): test of reliability and validity of the Danish version (SS-QOL-DK). Clin Rehabil 2007, 21:620-627.

19. Verbunt JA, Seelen HAM, Ramos FP, Michielsen BHM, Wetzelaer WL, Moennekens M: Mental practice-based rehabilitation training to improve arm function and daily activity performance in stroke patients: a randomized clinical trial. BMC Neurol 2008, 8:7.

20. Ewert T, Stucki G: Validity of the SS-QOL in Germany and in survivors of hemorrhagic or ischemic stroke. Neurorehabil Neural Repair 2007, 21:161-168.

21. Boosman H, Passier PECA, Visser-Meily JMA, Rinkel GJE, Post MWM: Validation of the Stroke-Specific Quality of Life Scale (SS-QOL) in patients with aneurysmal subarachnoid haemorrhage. J Neurol Neurosurg Psychiatry 2010, 81(5):485-9.

22. Teixeira-Salmela LF, Gomes-Neto M, Magalhaes LC, Lima RCM, Faria CDCM Content comparisons of stroke-specific quality of life based upon the international classification of functioning, disability, and health. Qual Life Res 2009, 18:765-773.

23. Portney LG, Watkins MP: Foundations of Clinical Research: Applications to Practice. 3 edition. Upper Saddle River, NJ: Pearson/Prentice Hall; 2009.

24. Lin $K C$, Fu T, Wu CY, et al: Minimal detectable change and clinically important difference of the Stroke Impact Scale in stroke patients. Neurorehabil Neural Repair 2010.

25. Plummer P, Behrman AL, Duncan PW, et al: Effects of stroke severity and training duration on locomotor recovery after stroke: a pilot study. Neurorehabil Neural Repair 2007, 21:137-151.

26. Evans MD, Goldie PA, Hill KD: Systematic and random error in repeated measurements of temporal and distance parameters of gait after stroke. Arch Phys Med Rehabil 1997, 78:725-729.

27. Perry J, Garrett M, Gronley JK, et al: Classification of walking handicap in the stroke population. Stroke 1995, 26:982-989.

28. Wu CY, Chuang LL, Lin KC, Chen HC, Tsay PW: Randomized trial of distributed constraint-induced therapy versus bilateral arm training for the rehabilitation of upper-limb motor control and function after stroke. Neurorehabil Neural Repair 2010.

29. Lin KC, Chang YF, Wu CY, Chen YA: Effects of constraint-induced therapy versus bilateral arm training on motor performance, daily functions, and quality of life in stroke survivors. Neurorehabil Neural Repair 2009, 23:441-448.

30. Bouffioulx E, Arnould C, Thonnard JL: A satisfaction measure of activities and participation in the actual environment experienced by patients with chronic stroke. J Rehabil Med 2008, 40:836-843.

31. Brunnstrom S: Movement Therapy in Hemiplegia New York, NY: Harper \& Row; 1970.

32. Folstein MF, Folstein SE, McHugh PR: "Mini-mental State." A practical method for grading the cognitive state of patients for the clinician. Psychiatr Res 1975, 12:189-198.

33. Bohannon R, Smith M: Interrater reliability of a modified Ashworth scale of muscle spasticity. Phys Ther 1987, 67:206-207.

34. Hsueh IP, Cheng CC, Jeng JS, Hsieh CL: Development of a scale measuring self-perceived difficulty in performing ADL for stroke patients. Proceedings of the Symposium of Occupational Therapy: 7 April 2005; Taipei 2005.

35. Visser-Meily JMA, Rhebergen ML, Rinkel GJE, van Zandvoort MJ, Post MWM: Long-term health-related quality of life after aneurysmal subarachnoid hemorrhage: Relationship with psychological symptoms and personality characteristics. Stroke 2009, 40:1526-1529.

36. Schmitt JS, Fabio RPD: Reliable change and minimum important difference (MID) proportions facilitated group responsiveness comparisons using individual threshold criteria. J Clin Epidemiol 2004, 57:1008-1018.

37. de Vet HCW, Terwee CB, Knol DL, Bouter LM: When to use agreement versus reliability measures. J Clin Epidemio 2006, 59:1033-1039.

38. Guyatt GH, Osoba D, Wu AW, et al: Methods to explain the clinical significance of health status measures. Mayo Clin Proc 2002, 77:371-383.

39. Cohen JW: Statistical Power Analysis for the Behavior Sciences. 2 edition. Hillsdale, NJ: Lawrence Erlbaum Associates; 1988.

40. Ringash J, O'Sullivan B, Bezjal A, Redelmeier DA: Interpreting clinically significant changes in patient-reported outcomes. Cancer 2007, 110:196-202.

41. Norman GR, Sloan JA, Wyrwich KW: Interpretation of changes in healthrelated quality of life: the remarkable universality of half a standard deviation. Med Care 2003, 41:582-592.

42. Lang CE, Edwards DF, Birkenmeier RL, Dromerick AW: Estimating minimal clinically important differences of upper-extremity measures early after stroke. Arch Phys Med Rehabil 2008, 89:1693-1700.

43. Cella D, Hahn EA, Dineen K: Meaningful change in cancer-specific quality of life scores: Differences between improvement and worsening. Qual Life Res 2002, 11:207-221.

44. Zisapel N, Nir T: Determination of the minimal clinically significant difference on a patient visual analog sleep quality scale. J Sleep Res 2003, 12:291-298.

45. Iyer LV, Haley SM, Watkins MP, Dumas HM: Establishing miniml clinically important differences for scores on the pediatric evaluation of disability inventory for inpatient rehabilitation. Phys Ther 2003, 83:888-898.

46. Hagg O, Fritzell P, Nordwall A: The clinical importance of changes in outcome scores after treatment for chronic low back pain. Eur Spine J 2003, 12:12-20. 
47. Duncan PW, Wallace D, Lai SM, Johnson D, Embretson S, Laster LJ: The Stroke Impact Scale version 2.0: evaluation of reliability, validity and sensitivity to change. Stroke 1999, 30:2131-2140.

48. Crosby RD, Kolotkin RL, Williams GR: Defining clinically meaningful change in health-related quality of life. J Clin Epidemiol 2003, 56:395-407.

49. Santanello NC, Zhang J, Seidenberg B, Reiss TF, Barber BL: What are minimal important changes for asthma measures in a clinical trial? Eur Respir J 1999, 14:23-27.

50. Revicki D, Hays RD, Cella D, Sloan J: Recommended methods for determining responsiveness and minimally important differences for patient-reported outcomes. J Clin Epidemiol 2008, 61:102-109.

51. Hsieh YW, Wang CH, Sheu CF, Hsueh IP, Hsieh CL: Estimating the minimal clinically important difference of the Stroke Rehabilitation Assessment of Movement measure. Neurorehabil Neural Repair 2008, 22:723-727.

52. Fritz SL, George SZ, Wolf SL, Light KE: Participant perception of recovery as criterion to establish important of improvement for constraintinduced movement therapy outcome measures: a preliminary study. Phys Ther 2007, 87:170-178.

53. Kupferberg DH, Kaplan RM, Slymen DJ, Ries AL: Minimal clinically important difference for the UCSD Shortness of Breath Questionnaire. J Cardiopulm Rehabil 2005, 25:370-377.

54. Wyrwich KW, Nienaber NA, Tierney WM, Wolinsky FD: Linking clinical relevance and statistical significance in evaluating intraindividual changes in health-related quality of life. Med Care 1999, 37:469-478.

55. Wyrwich KW, Tierney WM, Wolinsky FD: Further evidence supporting an SEM-based criterion for identifying meaningful intraindividual changes in health-related quality of life. J Clin Epidemiol 1999, 52:861-873.

56. Wyrwich KW, Tierney WM, Wolinsky FD: Using the standard error of measurement to identify important changes on the Asthma Quality of Life Questionnaire. Qual Life Res 2002, 11:1-7.

57. Jordan K, Dunn KM, Lewis M, Croft P: A minimal clinically important difference was derived for the Roland-Morris Disability Questionnaire for low back pain. J Clin Epidemiol 2006, 59:45-52.

58. Leidy NK, Wyrwich KW: Bridging the gap: Using triangulation methodology to estimate minimal clinically important differences (MCIDs). COPD 2005, 2:157-165.

59. Cella D, Bullinger M, Scott C, Barofsky I: Group vs. individual approaches to understanding the clinical significance of differences or changes in quality of life. Mayo Clin Proc 2002, 77:384-392.

60. Dawson J, Doll H, Coffey J, Jenkinson C: Responsiveness and minimally important change for the Manchester-Oxford foot questionnaire (MOXFQ) compared with AOFAS and SF-36 assessments following surgery for hallux valgus. Osteoarthritis Cartilage 2007, 15:918-931.

61. Resnik L, Dobrykowski E: Outcomes measurement for patients with low back pain. Orthop Nurs 2005, 24:14-24.

62. Norman G, Stratford P, Regehr G: Methodological problems in the retrospective computation of responsiveness to change: the lessons of Cronbach. J Clin Epidemiol 1997, 50:869-879.

63. Schwartz N, Sudman S: Autobiographical memory and the validity of retrospective reports New York: Springer-Verlag; 1994.

64. Davidson M, Keating JL: A comparison of five Low Back Disability Questionnaires: reliability and responsiveness. Phys Ther 2002, 82:8-24.

65. Barratt A, Wyer PC, Hatala R, et al: Tips for teachers of evidence-based medicine: 1 . Relative risk reduction, absolute risk reduction and number needed to treat. CMAJ 2004, 171:353-358.

66. Schmitt JS, Di Fabio RP: Reliable change and minimum important difference (MID) proportions facilitated group responsiveness comparisons using individual threshold criteria. J Clin Epidemiol 2004, 57:1008-1018

67. Faber J, Bosscher RJ, van Wieringen PC: Clinimetric properties of the performance-oriented mobility assessment. Phys Ther 2006, 86:944-954.

68. Twiss J, Doward LC, McKenna SP, Eckert B: Interpreting scores on multiple sclerosis-specific patient reported outcome measures (the PRIMUS and U-FIS). Health Qual Life Outcome 2010, 8:117.

69. Williams LS, Bakas T, Brizendine E, et al: How valid are family proxy assessments of stroke patients' health-related quality of life? Stroke 2006, 37:2081-2085.
70. Gundy CM, Aaronson NK: Effects of mode of administration (MOA) on the measurement properties of the EORTC QLQ-C30: a randomized study. Health Qual Life Outcomes 2010, 8:35.

doi:10.1186/1477-7525-9-5

Cite this article as: Lin et al:: Assessing the Stroke-Specific Quality of Life for Outcome Measurement in Stroke Rehabilitation: Minimal Detectable Change and Clinically Important Difference. Health and Quality of Life Outcomes 2011 9:5.

\section{Submit your next manuscript to BioMed Central and take full advantage of:}

- Convenient online submission

- Thorough peer review

- No space constraints or color figure charges

- Immediate publication on acceptance

- Inclusion in PubMed, CAS, Scopus and Google Scholar

- Research which is freely available for redistribution

Submit your manuscript at www.biomedcentral.com/submit
Biomed Central 